\title{
ANÁLISE DE OSTEOBLASTOS HUMANOS CULTIVADOS SOBRE NANOFIBRAS POLIMÉRICAS RECOBERTAS COM GELATINA
}

\author{
Mayara Cristina Moreira Silva ${ }^{1}$ \\ Fernanda Roberta Marciano² \\ Maíra Maftoum Costa ${ }^{3}$ \\ Anderson de Oliveira Lobo ${ }^{4}$
}

\begin{abstract}
Resumo: Os índices de doenças relacionadas às estruturas ósseas têm aumentado nas últimas décadas assim como a necessidade de se desenvolver novos biomaterias e melhorar os já existentes tem aumentado. O objetivo deste estudo foi recobrir nanofibras poliméricas com gelatina e analisar células de osteoblastos humanos (MG-63) cultivados sobre este biomaterial. Foram verificadas a toxicidade das mantas poliméricas e a proliferação celular dos osteoblastos cultivados utilizando o ensaio de LDH (lactato desidrogenasse). As nanofíbras poliméricas foram produzidas pela eletrofiação de solução de poli (butileno adipato cotereftalo) (PBAT) a $12 \% \mathrm{w} / \mathrm{V}$ com nanohidroxiapatita ( $\mathrm{nHAp}$ ) a $3 \% \mathrm{w} / \mathrm{v}$ e recobertas com gelatina (GE) a $5 \%$ w/v. A presença da GE foi confirmada por microscopia eletrônica de varredura e a não toxicidade das fibras após o período de 24 h pela mensuração de LDH liberado no meio. A proliferação celular após 7 e 14 dias não sofreu alterações quando comparado com o controle. Os resultados obtidos são promissores e sugerem o recobrimento das nanofibras como uma alternativa viável para aumentar a hidrofilicidade de materiais sem interferir na toxicidade do mesmo.
\end{abstract}

Palavras-chave: Nanofibras poliméricas; PBAT; nHAp; Gelatina; Osteoblástos.

\footnotetext{
1 Laboratório de Nanotecnologia Biomédica/Universidade do Vale do Paraíba, Brasil. E-mail: mayaramoreira.mm@gmail.com.

2 Laboratório de Nanotecnologia Biomédica/Universidade do Vale do Paraíba, Brasil. E-mail: frmarciano@univap.br.

3 Laboratório de Nanotecnologia Biomédica/Universidade do Vale do Paraíba, Brasil. E-mail: maftoum@gmail.com.

4 Laboratório de Nanotecnologia Biomédica/Universidade do Vale do Paraíba, Brasil. E-mail: lobo.aol@gmail.com.
} 